\title{
Contaminantes y aprovechamiento de desechos en curtidoras de León, producir biogás y biodiesel
}

\section{Pollutants and use of waste in lion tanning, producing biogas and biodiesel}

\author{
Martínez Cubillo, Francisco Javier; Zuniga-Gonzalez, Editor \\ Academcio Prof. Dr. Carlos Alberto
}

Francisco Javier Martínez Cubillo

francisco.martinez@curj.unanleon.edu.ni

Universidad Nacional Autónoma de Nicaragua, León,

CUR-Jinotega, Nicaragua

Editor Academcio Prof. Dr. Carlos Alberto Zuniga-

Gonzalez

Universidad Nacional Autónoma de Nicaragua,

León. Escuela de Ciencias Agrarias y Veterinarias.

Departamento de Agroecología. , Nicaragua

Revista Iberoamericana de Bioeconomía y Cambio

Climático

Universidad Nacional Autónoma de Nicaragua, León, Nicaragua

ISSN-e: 2410-7980

Periodicidad: Semestral

vol. 4, núm. 8,2018

czuniga@ev.unanleon.edu.ni

Recepción: 15 Mayo 2018

Aprobación: 15 Octubre 2018

URL: http://portal.amelica.org/ameli

atsRepo/394/3941755006/index.htm

DOI: https://doi.org/10.5377/ribcc.v4i8.6675

Autor de correspondencia:

francisco.martinez@curj.unanleon.edu.ni
Resumen: Los mataderos y curtidoras de cueros causangran contaminación ambiental, principalmente por el cromo trivalente y a veces hexavalente, la sangre, grasas y desechos orgánicos que se descomponen. En León, los mataderos y curtidoras, no siguen los lineamientos para el tratamiento de residuos y reducción de la contaminación. Con este trabajo se busco hacer un estudio de los contaminantes ambientales en las industrias mataderos y curtidoras, realizando mediciones ambientales fisicoquímicas para verificar el impacto ambiental. Se construyó un biodigestor y un mini reactor para obtener de biogás y biodiesel, usando materia prima los residuos, tanto orgánicos, como grasos, como fuente de aprovechamiento y reducción de la contaminación. Se dividió en 4 zonas para el análisis, los parámetros fisicoquímicos medidos fueron: $\mathrm{pH}$, $\mathrm{CO}_{2}(\mathrm{ppm}), \mathrm{O}_{2}$ disuelto $(\mathrm{mg} / \mathrm{L})$, Conductividad $(\mu \mathrm{s} / \mathrm{cm})$, DBO5, DQO, color, olor, aspecto, temperatura, humedad relativa.Según la relación $\mathrm{DBO} 5 / \mathrm{DQO}$, las 4 zonas están encima de 8 , esto indica que el agua es más biodegradable y puede ser tratada biológicamente para su limpieza, los niveles de $\mathrm{CO}_{2}$, indican que son adecuados para zonas de ríos, lagos, lagunas y mares, sobre todo donde existe flora que pueda utilizar el $\mathrm{CO}_{2}$. Todos los desechos orgánicos son aprovechables para obtener de biogás (metano).

Palabras clave: Contaminación, Medio ambiente, Mataderoscurtidoras, Biogas, Biodiesel.

Abstract: Slaughterhouses and leather tanners cause great environmental contamination, mainly by trivalent chromium and sometimes hexavalent, blood, fats and organic waste that decompose. In León, slaughterhouses and tanneries do not follow the guidelines for waste treatment and pollution reduction. With this work we seek to make a study of environmental contaminants in slaughterhouses and tanning industries, making physical- chemical environmental measurements to verify the environmental impact. A biodigester and a mini reactor were built to obtain biogas and biodiesel, using both organic and fatty waste as raw material, as a source of use and reduction of pollution. was divided into 4 zones for analysis, the physicochemical parameters measured were: $\mathrm{pH}$, $\mathrm{CO}_{2}(\mathrm{ppm})$, dissolved $\mathrm{O}_{2}(\mathrm{mg} / \mathrm{L})$, Conductivity $(\mu \mathrm{s} / \mathrm{cm})$, BOD5, COD, color, smell, appearance, temperature, humidity relative. According to the $\mathrm{BOD} 5 / \mathrm{COD}$ ratio, the 4 zones 
are above 8 , this indicates that the water is more biodegradable and can be treated biologically for cleaning, $\mathrm{CO}_{2}$ levels indicate that they are suitable for river, lake, lagoon and river areas. seas, especially where there is flora that can use $\mathrm{CO}_{2}$. All organic waste can be used to obtain biogas (methane).

Keywords: Pollution, environment, slaughterhouses-tanning, Biogas, Biodiesel.

\section{INTRODUCCIÓN}

La ciudad de león está ubicada latitud $12^{\circ} 26.27^{\prime}$ y longitud $86^{\circ} 52.68^{\prime}$. Las empresas se ubican en la zona sur oeste, colinda con los barrios subtiava y laborío y a orillas del rio chiquito. Esta ubicación hace que la contaminación del rio sea notable y un tema importante ya que la población además de contaminarlo con basura y desechos hace uso de sus aguas para diversas labores, lo que conlleva a una cadena de contaminación.

Los mataderos ycurtidoras generan grandes cantidades de aguas residuales tanto en estado gaseoso, líquido o sólido, con alto contenido de materia orgánica. Estos a través de procesos químicos o uso para generar gas, combustible y energía, pueden ser separados de las aguas y reutilizados de nuevo en el proceso (Artuz, et al., 2011), (Tablero, H., 2006). En el caso de mataderos los contaminantes son: sangre, grasas y el mal olor por la descomposición orgánica, que constituye una molestia constante. En el proceso de curtido, se generan lodos si hay planta de tratamiento y muchos contaminantes de cromo si no hay (Cado, 1996). El índice de descarga varía desde 14 hasta $116 \mathrm{~L}$ de aguas residuales por kg de materia prima (Palacios y Zapata, 2011). Los mataderos se rigen por la necesidad alimenticia y el curtido de pieles esuna actividad estrechamente ligada a sectoresproductivos como la industria del calzado. Estas industriasestán fuertemente establecidas en la ciudad de León, Nicaragua, habiendo aproximadamente 3 mataderos y 23 curtidoras (Reconocidas), hay aproximadamente quince teneros trasladados a donde fue construido el Complejo industrial de teneros a doscientos metros de "la Chanchera", carretera a Poneloya los otros aún se oponen a dejar el lugar donde tradicionalmente han funcionado. Más del $90 \%$ utilizan técnicas artesanales y sin ningún método para tratar sus desechos.

Se han realizado varios estudios, en 1997 y 2011, por la UNAN-León, en el área de Biología y química respectivamente, con lo que Salmerón y Rodríguez (1997) y Palacios y Zapata (2011), plantean que el rio está en una situación muy grave, debido a la permanente contaminación de aguas residuales y el vertido de desechos de empresas (curtidoras), la deforestación, vertido de aguas negras y pluviales, que según el índice Simplificado de calidad de Agua (ISCA) y las normas CAPRE, el agua es pésima y mala calidad en todo el recorrido urbano y utilizable con buena calidad en el sector rural. Con este trabajo se pretende generar conciencia de la contaminación y soluciones prácticas, sencillas y rápidas, además darles un uso importante a los desechos en dos aspectos:

- Convirtiendo desechos sólidos orgánicos en biogás que podrían ser utilizado en la industria, con la construcción de un biodigestor y/o utilizar la grasa de desecho para obtener biodiesel, fabricando un mini reactor, con materiales caseros,

- Verificar la funcionalidad de la planta de tratamiento de desechos líquidos de las empresas que las posean y recomendar mejoras o soluciones los desechos que vierten al rio.

\section{NotAS DE AUTOR}


Estos aspectos mencionados son fácilmente aplicables con tecnologías sencillas y sobre todo baratas, además se tratará de aprovechar al máximo los desechos y reducir la contaminación (Rivera et al. 2009; Mata Chasi, 2010).

\section{Materiales y MÉtodos}

El estudio es de tipo descriptivo y prospectivo. Se realizó por medio de un análisisobservacional, como experimental, realizándose de la siguiente manera:

- Visitas a las empresas para conocer su sistema de trabajo, instalaciones, maquinaria en uso, tipos de desechos, tratamiento de desechos y donde se desechan.

- Visitas al Rio Chiquito para tomar muestras y realizar análisis directos, desde el puente Vallecas hasta 100 metros rio abajo (oeste ciudad), después del desecho de el rastro municipal

- Las zonas de análisis de Rio chiquito se dividieron en 4 zonas, constituidas por:

Zona 1: puente Vallecas realizados dentro de la empresa como en las zonas del rio

Zona 2: Curtidora 1

Zona 3: Curtidora 2

Zona 4: Curtidora 3 y desechos de rastro municipal

- Análisis fisicoquímicos básicos.

- Los análisis realizados tanto dentro de la empresa como en el rio chiquito se llevaron a cabo mediante la Interface LabQuest los análisis de: $\mathrm{pH}, \mathrm{O}_{2}$ disuelto, Conductividad, $\mathrm{CO}_{2}$,Turbidez. Los análisis de $\mathrm{DBO} 5$ y DQO se realizaron en el laboratorio (Figura 1).

- Muestrear los diferentes desechos para realizarpruebas en el laboratorio de obtención de: biogás y biodiesel, a través de (biodigestor y reactor)(Figura 9; Figura 10), que a la vez puedan utilizar el producto en sus procesos diarios.
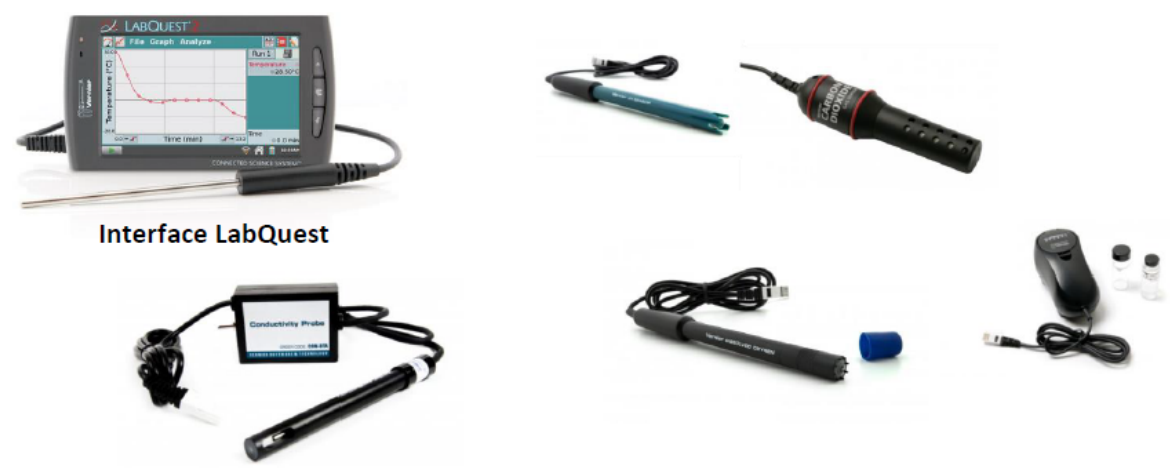

FIGURA 1.

Accesorios de la Interface LabQuest

\section{Resultados y Discusión}

Los resultados de la investigacion evidencian el nivel de $\mathrm{pH}, \mathrm{CO} 2, \mathrm{DBO}, \mathrm{DQO}$, Conductividad y Turbidez ante los contaminantes en el agua por desechos del proceso dentro de las empresas objetos de estudio (Figura 2) 
Francisco Javier Martínez Cubillo, et al. Contaminantes y aprovechamiento de desechos en Curtidora...

\begin{tabular}{|c|c|c|c|c|c|c|c|c|}
\hline & $\mathrm{pH}$ & $\begin{array}{c}\mathrm{CO}_{2} \\
\text { (ppm) }\end{array}$ & $\begin{array}{c}\mathrm{DBO}_{5} \\
(\mathrm{mg} / \mathrm{L})\end{array}$ & $\begin{array}{c}\text { DQO } \\
(\mathrm{mg} / \mathrm{L})\end{array}$ & $\begin{array}{l}\text { CONDUCTIVI } \\
\text { DAD }(\mu \mathrm{s} / \mathrm{cm})\end{array}$ & $\begin{array}{l}\text { TURBDEZ } \\
\text { (NTU) }\end{array}$ & $\begin{array}{c}\text { Humedad } \\
\text { Relativa } \\
\%\end{array}$ & $\begin{array}{c}\text { O: DISUELTO } \\
(\mathrm{mg} / \mathrm{L})\end{array}$ \\
\hline PROMEDIO & 6,70 & 1471,10 & 50,98 & 4,22 & 232,67 & 20,16 & 61,13 & 0,78 \\
\hline DESVEST & 0,086 & 21,08 & 0,12 & 0,17 & 0,88 & 0,15 & 0,96 & 0,02 \\
\hline ERROR & 0,003 & 0,003 & 0,0006 & 0,010 & 0,0009 & 0,001 & 0,003 & 0,008 \\
\hline
\end{tabular}

\begin{tabular}{|c|c|c|c|c|c|c|c|c|}
\hline & $\mathrm{pH}$ & $\begin{array}{l}\mathrm{CO}_{2} \\
(\mathrm{ppm})\end{array}$ & $\begin{array}{c}\mathrm{DBO}_{5} \\
(\mathrm{mg} / \mathrm{L})\end{array}$ & $\begin{array}{c}\text { DQO } \\
(\mathrm{mg} / \mathrm{L})\end{array}$ & $\begin{array}{c}\text { CONDUCTIVID } \\
\text { AD }(\mu \mathrm{s} / \mathrm{cm})\end{array}$ & $\begin{array}{l}\text { TURBIDEZ } \\
\text { (NTU) }\end{array}$ & $\begin{array}{c}\text { Humedad } \\
\text { Relativa } \\
\%\end{array}$ & $\mathrm{O}_{2} \underset{(\mathrm{mg} / \mathrm{L})}{\text { DISUELTO }}$ \\
\hline PROMEDIO & 5,69 & 1520,00 & 50,48 & 5,80 & 234,42 & 81,51 & 61,98 & 0,78 \\
\hline DESVEST & 0,21 & 98,06 & 0,16 & 0,10954 & 0,57 & 0,50 & 1,20 & 0,02 \\
\hline ERROR & 0,009 & 0,016 & 0,0008 & 0,004 & 0,0006 & 0,002 & 0,005 & 0,008 \\
\hline
\end{tabular}
ZONA 3

\begin{tabular}{|c|c|c|c|c|c|c|c|c|}
\hline & $\mathrm{pH}$ & $\begin{array}{c}\mathrm{CO}_{2} \\
(\mathrm{ppm})\end{array}$ & $\begin{array}{c}\mathrm{DBO}_{\theta} \\
(\mathrm{mg} / \mathrm{L})\end{array}$ & $\begin{array}{c}\mathrm{DQO} \\
(\mathrm{mg} / \mathrm{L})\end{array}$ & $\begin{array}{c}\text { CONDUCTIVID } \\
\text { AD }(\mu \mathrm{s} / \mathrm{cm})\end{array}$ & $\begin{array}{l}\text { TURBIDEZ } \\
\text { (NTU) }\end{array}$ & $\begin{array}{c}\text { Humedad } \\
\text { Relativa } \\
\% \\
\end{array}$ & $\begin{array}{c}\mathrm{O}_{2} \text { DISLELTO } \\
(\mathrm{mg} / \mathrm{L})\end{array}$ \\
\hline PROMEDIO & 6,46 & 1598,50 & 47,65 & 5,42 & 240,37 & 52,94 & 60,10 & 0,78 \\
\hline DESVEST & 0,21 & 98,06 & 0,16598 & 0,10954 & 0,57 & 0,50 & 1,20 & 0,02 \\
\hline ERROR & 0,002 & 0,031 & 0,0006 & 0,004 & 0,001 & 0,0007 & 0,011 & 0,012 \\
\hline
\end{tabular}

ZONA 4

\begin{tabular}{|c|c|c|c|c|c|c|c|c|}
\cline { 2 - 9 } \multicolumn{1}{c|}{} & $\mathbf{p H}$ & $\begin{array}{c}\mathbf{C O}_{2} \\
(\mathbf{p p m})\end{array}$ & $\begin{array}{c}\mathbf{D B O} \\
(\mathbf{m g} / \mathbf{L})\end{array}$ & $\begin{array}{c}\mathbf{D Q O} \\
(\mathbf{m g} / \mathbf{L})\end{array}$ & $\begin{array}{c}\text { CONDUCTIVID } \\
\mathbf{A D}(\mathbf{\mu s} / \mathbf{c m})\end{array}$ & $\begin{array}{c}\text { TURBIDEZ } \\
(\mathbf{N T U})\end{array}$ & $\begin{array}{c}\text { Humedad } \\
\text { Relativa } \\
\mathbf{\%}\end{array}$ & $\begin{array}{c}\mathbf{O}_{2} \text { DISLELTO } \\
(\mathbf{m g} / \mathbf{L})\end{array}$ \\
\hline PROMEDIO & 6,65 & 1564,40 & 49,48 & 6,08 & 238,00 & 95,18 & 62,75 & 0,80 \\
\hline DESVEST & 0,13 & 22,54 & 0,12 & 0,13 & 0,32 & 1,01 & 0,88 & 0,03 \\
\hline ERROR & 0,005 & 0,004 & 0,0006 & 0,006 & 0,0003 & 0,003 & 0,004 & 0,0099 \\
\hline
\end{tabular}

FIGURA 2.

Contaminantes de agua por desechos de proceso vertidos al rio Chiquito

Los resultados muestran las mediciones diarias de contaminantes de agua por desechos de proceso dentro de la empresa, fueron medidas variables como $\mathrm{pH}, \mathrm{CO}_{2}$, Conductividad y $\mathrm{O}_{2}$ (Figura 3, Figura 4, Figura 5, Figura 6).

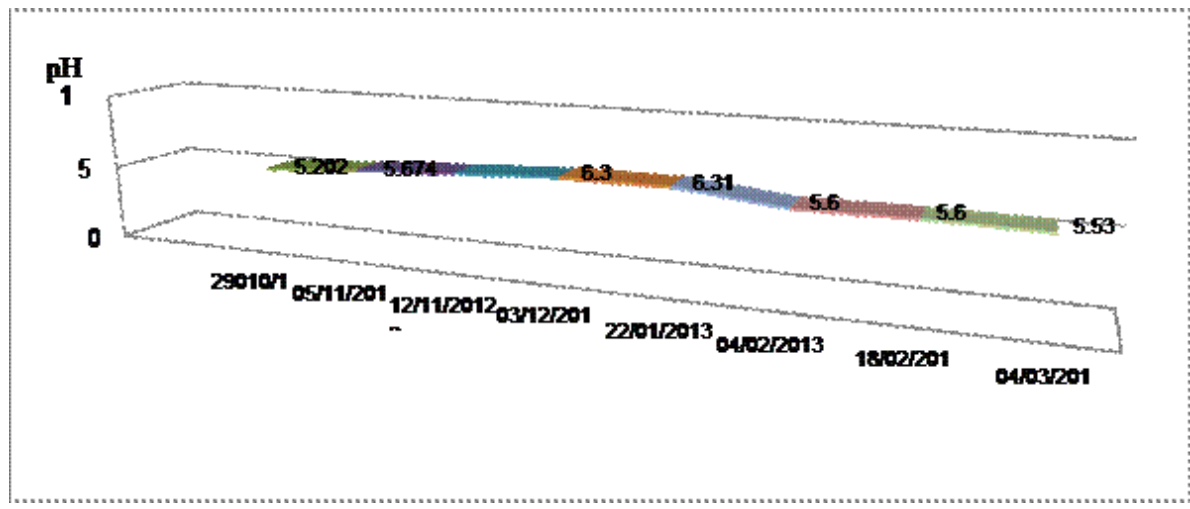

FIGURA 3

Promedio de $\mathrm{pH}$ por día de análisis, zona agua LACA, dentro empresa 


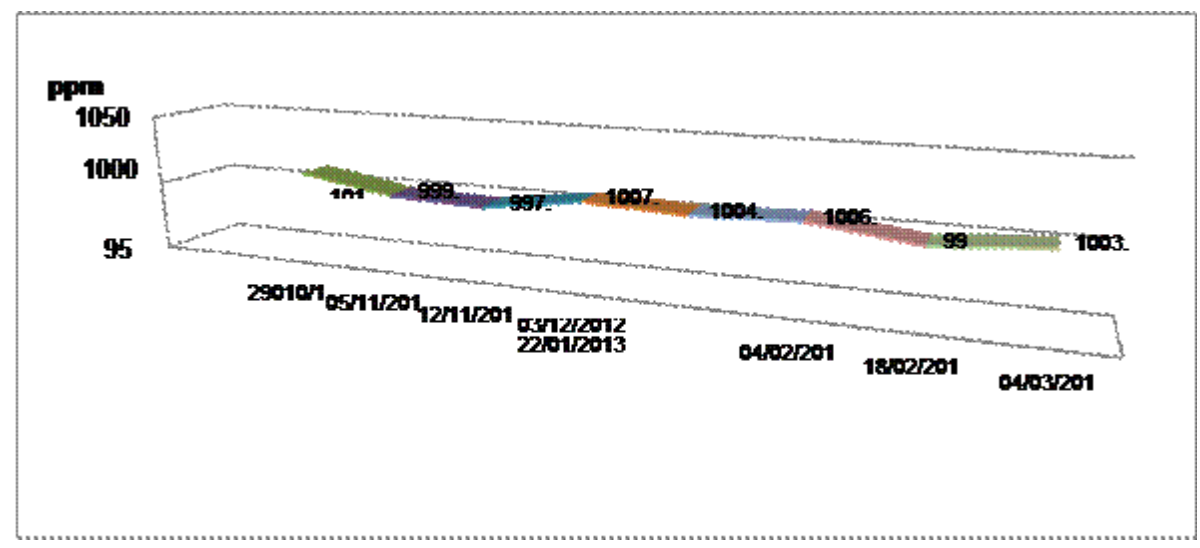

FIGURA 4

Promedio $\mathrm{CO}_{2}$ por dia de analisis zona agua LACA, dentro empresa

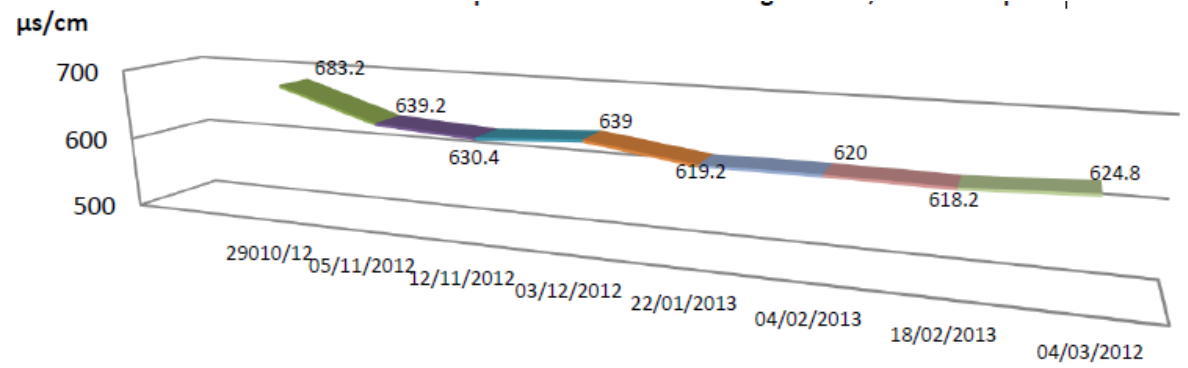

FIGURA 5.

Promedio Conductividad por dia de analisis zona agua LACA, dentro empresa

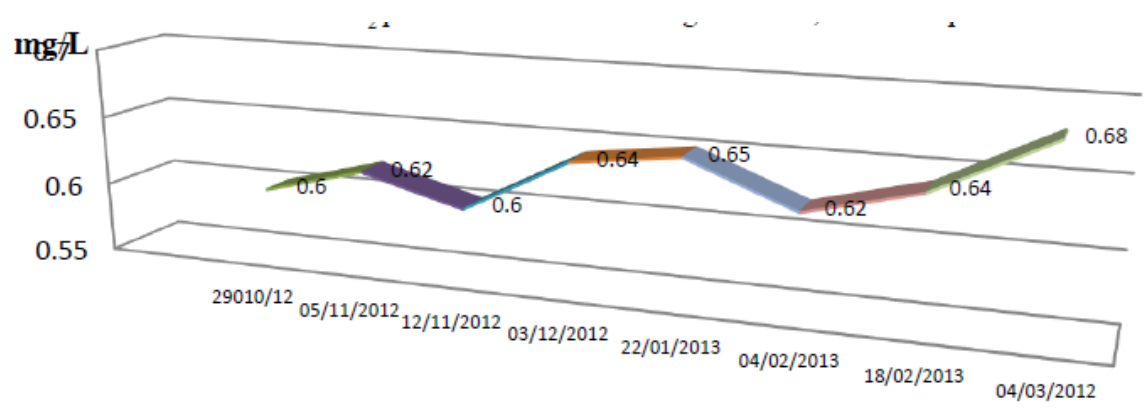

FIGURA 6.

Promedio $\mathrm{O}_{2}$ por dia de analisis zona agua LACA, dentro empresa

En la figura 7 se presenta la relación entre contaminantes en las 4 zonas analizadas a lo largo del rio chiquito. 


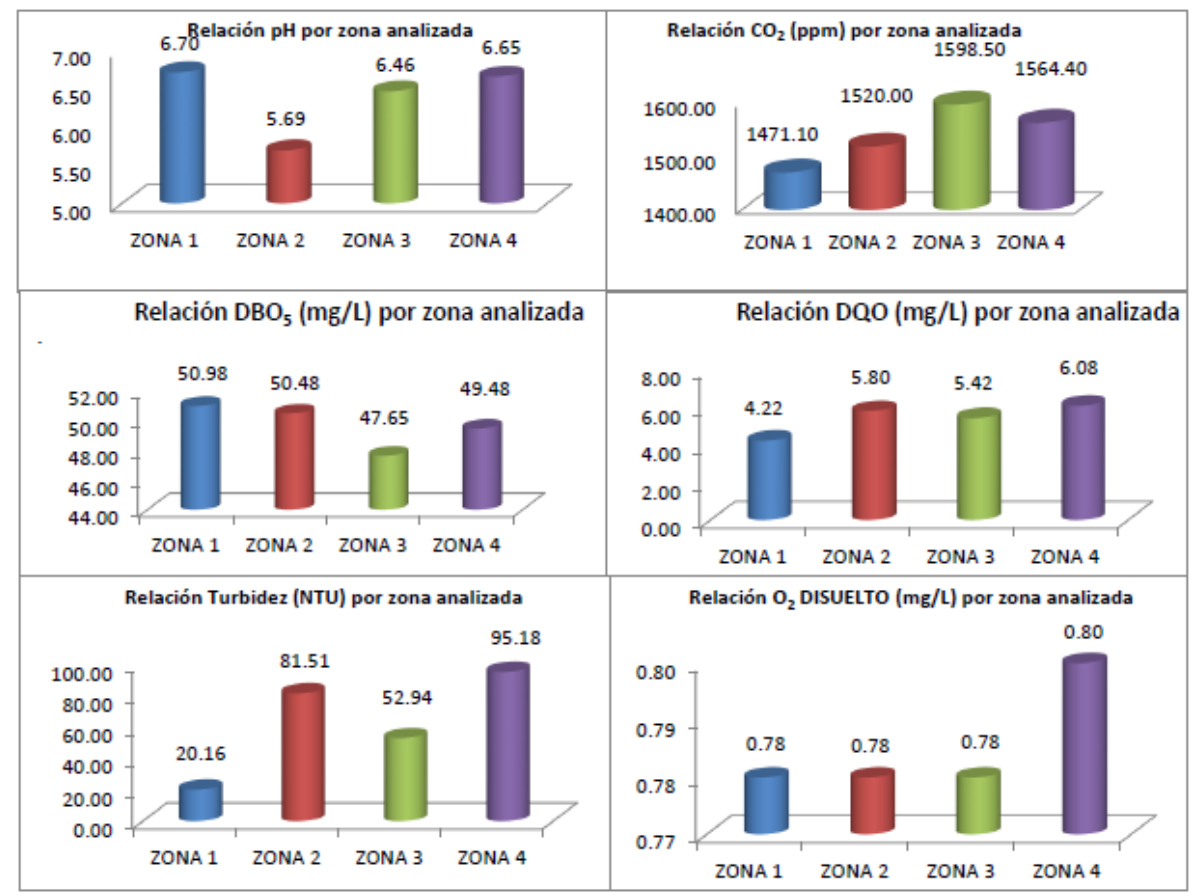

FIGURA 7

Relación entre contaminantes en las 4 zonas analizadas a lo largo del rio chiquito

En el análisis físico visual de la contaminación del rio se observo de aspecto turbio, con olor desagradable fuerte a huevo podrido tiene un color plomo debido al cromo desechado (Figura 8).

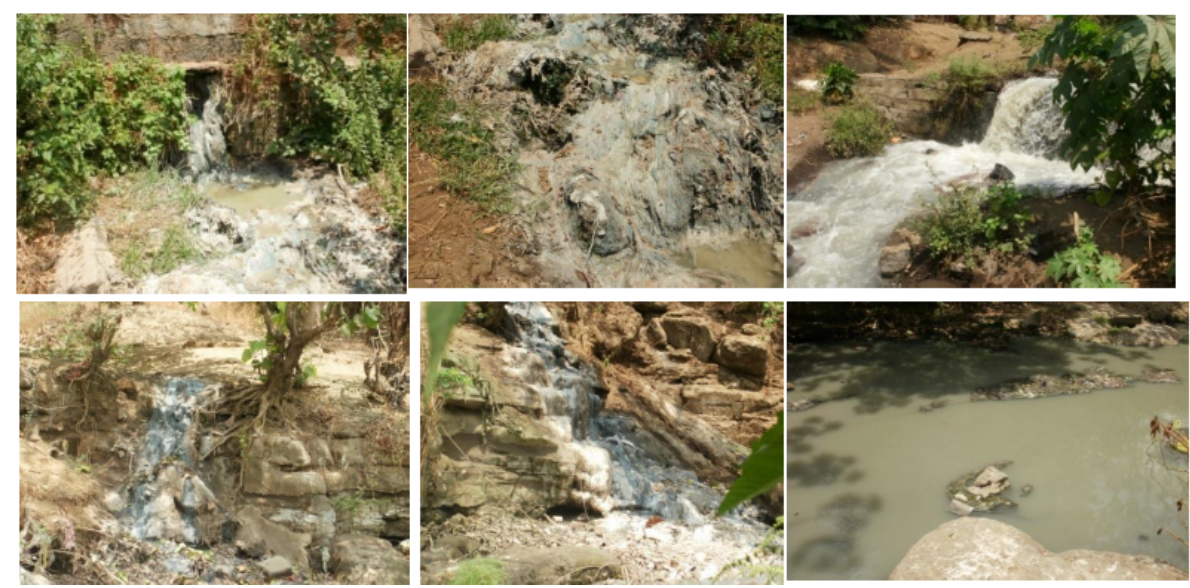

FIGURA 8.

Análisis físico visual de la contaminación del rio (color, olor, aspecto) 


\section{Construcción de equipos}
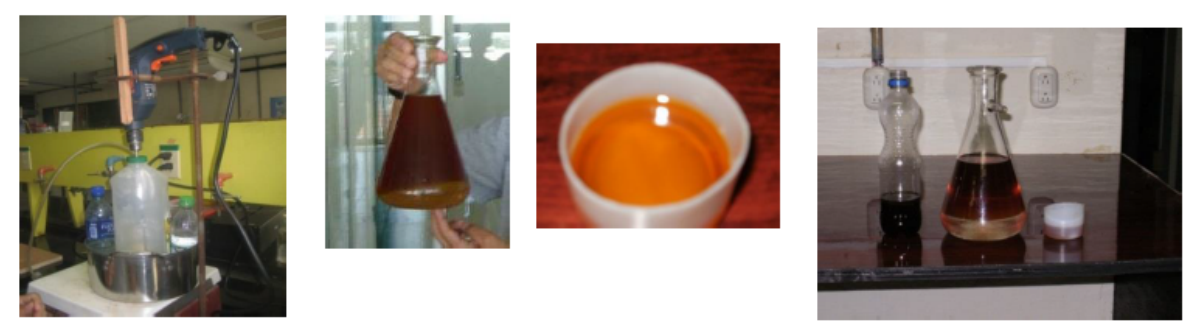

FIGURA 9

Mini reactor para obtención de biodiesel

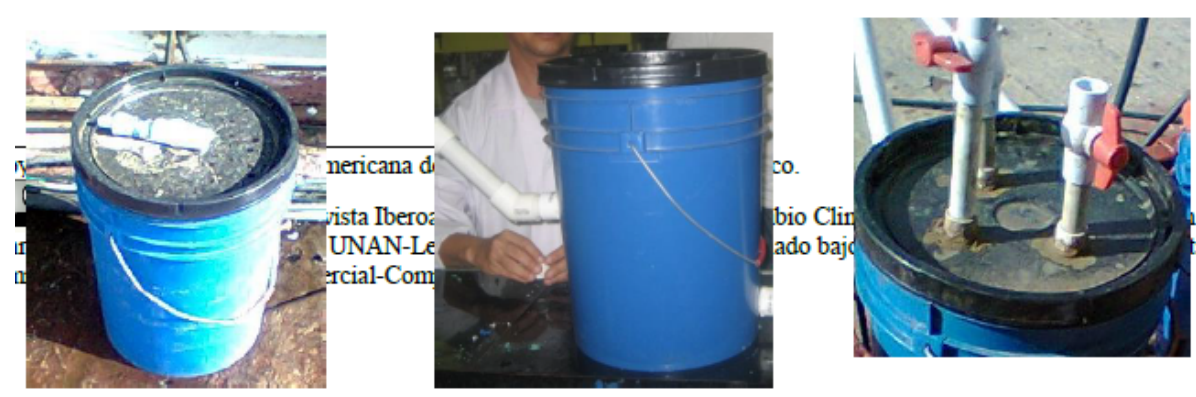

FIGURA 10

Biodigestor para obtención de biogas

\section{Conclusiones}

- La industria de cuero y matarifes generan gran cantidad de materia orgánica y aguas residuales como desecho, incidiendo principalmente en la contaminación del Rio chiquito y la población a sus alrededores.

- Estos contaminantes en su mayoría provenientes de la industria artesanal de cuero: no poseen ningún tratamiento de desechos y ningún control por parte de las entidades correspondientes.

- Sólo hay una empresa curtidora que posee planta de tratamiento y ha renovado su proceso y controles para disminuir al máximo la contaminación ambiental y adecuarse a las normas establecidas.

- Los análisis químicos indican que existe contaminación por: vertidos caseros, aguas negras, desechos de curtidoras y mataderos. En las curtidoras la contaminación aumenta con la cantidad de sólidos disueltos, el pH y la insuficiente cantidad de $\mathrm{O}_{2}$ para la supervivencia de especies marinas.

- Según la relación DBO5/DQO, las 4 zonas están encima de 8, esto indica que el agua es más biodegradable y puede ser tratada biológicamente para su limpieza, si los niveles son menores a 0.2 ó 0.5 indican que el agua no es tan biodegradable y que el tratamiento para su saneamiento seria más severo y químico.

- Los niveles de $\mathrm{CO}_{2}$, indican que son adecuados para zonas de ríos, lagos, lagunas y mares, sobre todo donde existe flora que pueda utilizar el $\mathrm{CO}_{2}$.

- Todos los desechos orgánicos son aprovechables para obtener de biogás (metano), usado en cocinas o para calentar agua en la misma empresa. Se obtuvieron en promedio $0.12 \mathrm{~L}\left(0.00012 \mathrm{~m}^{3}\right)$ de biogás al día, en un biodigestor de 15 litros de materia. En un matadero se podría obtener un promedio de $750 \mathrm{~m}^{3}$ de gas diario y $18,780 \mathrm{~m}^{3}$ mensual, si con una producción de $0.2 \mathrm{~m}^{3}$ se puede cocinar entre 2-3 horas la producción obtenida de gas seria de mucha importancia.

- Los desechos grasos se pueden utilizan para producción de biodiesel, utilizado en cualquier equipo diesel.

- Se obtiene entre un $65-70 \%$ de biodiesel a partir de las grasas, y un $75-80 \%$ si se utilizan aceites, con otro residuo como es la glicerina que puede tener un valor comercial. 
Francisco Javier Martínez Cubillo, et al. Contaminantes y aprovechamiento de desechos en Curtidora...

\section{LITERTURA CITADA}

ARTUZ, L. A., MARTÍNEZ, M. S., \& MORALES, C. J. (2011). LAS INDUSTRIAS CURTIEMBRES Y SU INCIDENCIA EN LA CONTAMINACIÓN DEL RÍO BOGOTÁ. ISOCUANTA, 1(1).

CADO, O. (1996). SALES DE CROMO: SU RELACIÓN CON EL MEDIO AMBIENTE. GERENCIA AMBIENTAL, 3(30), $770-778$.

Tablero, H. (2006). Medio ambiente. Recuperado de http://www. monografias. com/trabajos15/ medioambientevenezuela/medioa mbiente-venezuela. shtml.

Mata Chasi, E. A. (2010). Elaboración de una guía práctica para el manejo de los desechos sólidos y su incidencia en la contaminación ambiental en la ciudad de Saquisili, barrió la compañia durante el periodo 2009 - 2010. (Tesis de pregrado) Unidad Académica de Ciencias Administrativas y Humanísticas. UTC. Latacunga.

Palacios, H., \& Zapata, J. (2011). Estimación del indice simplificado de la calidad de agua (ISCA) del rio Chiquito de la ciudad de León. (Doctoral dissertation, Tesis de pregrado) UNAN-León, León, Nicaragua.

Rivera, I; Villanueva, G. \& Sandoval, G. (2009). Producción de biodiesel a partir de residuos grasos animales por vía enzimática. GRASAS Y ACEITES, 60 (5) 468-474.

Salmerón, A., \& Rodríguez, L. (1997). Análisis de la contaminación microbiana por patógenos en el rio chiquito y rio la gallina. (Doctoral dissertation, Tesis de pregrado), UNAN-León. León, Nicaragua. 